\title{
CORRECTION
}

\section{Correction to: Glucerabacter canisensis gen. nov., sp. nov., isolated from dog feces and its effect on the hydrolysis of plant glucosylceramide in the intestine of dogs}

\author{
Misho Kawata ${ }^{1} \cdot$ Ami Tsukamoto $^{1} \cdot$ Ryohei Isozaki $^{1} \cdot$ Shohei Nobukawa $^{1} \cdot$ Natsuki Kawahara $^{1} \cdot$ Shoko Akutsu $^{1}$. \\ Masato Suzuki ${ }^{1} \cdot$ Narito Asanuma ${ }^{1}$
}

Published online: 7 September 2021

(c) Springer-Verlag GmbH Germany, part of Springer Nature 2021

\section{Correction to: Archives of Microbiology (2018) 200:505-515 https://doi.org/10.1007/s00203-017-1463-1}

There are few corrections in the original publication. The detail is given below.

Original P.505 L.1 (In title)

\section{Glucerabacter canisensis}

Correction

\section{Gluceribacter canis}

Original P.505 L.15 (In Abstract)

Glucerabacter canisensis

Correction

Gluceribacter canis

Original P.505 L.20 (In Keywords)

Glucerabacter canisensis

Correction

The original article can be found online at https://doi.org/10.1007/ s00203-017-1463-1.

Narito Asanuma

asanuma@meiji.ac.jp

1 Department of Life Science, Meiji University, Higashimita, Tama-ku, Kawasaki 214-8571, Japan
Gluceribacter canis

Original P.505 Left paragraph L.33

Glucerabacter canisensis

Correction

Gluceribacter canis

Original P.506 Left paragraph L.27

Glucerabacter canisensis

Correction

Gluceribacter canis

Original P.512 Left paragraph L.4

Glucerabacter canisensis

Correction

Gluceribacter canis

Original P.513 Right paragraph L.1

Description of Glucerabacter gen. nov.

Glucerabacter (glu.cera.bac'ter. N.L. gen. n. glucera, GluCer; N.L. masc. n. bacter a rod; N.L. masc. n. Glucerbacter, a GluCer-utilizing rod).

Correction 


\section{Description of Gluceribacter gen. nov.}

Gluceribacter (Glu.ce.ri.bac.ter'. N.L. neut. n. glucerum GluCer; N.L. masc. n. bacter a rod; N.L. masc. n. Gluceribacter, a GluCer-utilizing rod).

Original P.1513 Right paragraph L.11

The genus is a member of the family Lachnospiraceae.

\section{Correction}

The genus is a member of the family Lachnospiraceae. The type species is "Gluceribacter canis".

Original P.513 Right paragraph L.12

\section{Description of Glucerabacter canisensis sp. nov.}

Glucerabacter canisensis (canis.ensis. N. masc. canis, dog; L. masc. ensis, from; N.L. masc. n. canisensis, from a dog)

Correction

\section{Description of Gluceribacter canis sp. nov.}

Gluceribacter canis (ca'nis. L. gen. n. canis of a dog)

Original P.514 Left paragraph L.2

Whole-cell fatty acids are $\mathrm{C}_{12: 0}, \mathrm{C}_{14: 0}, \mathrm{C}_{14: 0} \mathrm{DMA}$, unknown fatty acid with equivalent length $14.762 / \mathrm{C}_{15: 2} / \mathrm{C}_{15: 1}, \mathrm{C}_{16: 0}$ aldehyde, $\mathrm{C}_{16: 1}$ w7c, $\mathrm{C}_{16: 0}, \mathrm{C}_{16: 1}$ w7c DMA, C $\mathrm{C}_{16: 0}$ DMA, $\mathrm{C}_{17: 1} \mathrm{w} 8 \mathrm{c} / \mathrm{C}_{17: 2}, \mathrm{C}_{18: 1} \mathrm{w} 9 \mathrm{c}, \mathrm{C}_{18: 1}$ w7c/unknown fatty acid with equivalent length $17.834, \mathrm{C}_{18: 0}, \mathrm{C}_{18: 1}$ w9c DMA, $\mathrm{C}_{18: 1}$ w7c DMA, and $\mathrm{C}_{18: 0}$ DMA (representing more than 1.0\%).

\section{Correction}

Whole-cell fatty acids are $\mathrm{C}_{12: 0}, \mathrm{C}_{14: 0}, \mathrm{C}_{14: 0} \mathrm{DMA}$, unknown fatty acid with equivalent length $14.762 / \mathrm{C}_{15: 2} / \mathrm{C}_{15: 1}, \mathrm{C}_{16: 0}$ aldehyde, $\mathrm{C}_{16: 1} \omega 7 \mathrm{c}, \mathrm{C}_{16: 0}, \mathrm{C}_{16: 1} \omega 7 \mathrm{c}$ DMA, $\mathrm{C}_{16: 0}$ DMA, $\mathrm{C}_{17: 1} \omega 8 \mathrm{c} / \mathrm{C}_{17: 2}, \mathrm{C}_{18: 1} \omega 9 \mathrm{c}, \mathrm{C}_{18: 1} \omega 7 \mathrm{c} /$ unknown fatty acid with equivalent length $17.834, \mathrm{C}_{18: 0}, \mathrm{C}_{18: 1} \omega 9 \mathrm{c}$ DMA, $\mathrm{C}_{18: 1}$ $\omega 7 \mathrm{c}$ DMA, and $\mathrm{C}_{18: 0}$ DMA (representing more than $1.0 \%$ ).

Original P.514 Left paragraph L.9

$\operatorname{JCM} 31739^{\mathrm{T}}$

\section{Correction}

$\operatorname{JCM} 31739^{\mathrm{T}}$

\section{Original Table 2}

Line 4, Column 2 (Line; Spore formation, Column; Characteristic 1)

$+$

Correction

Line 4, Column 2 (Line; Spore formation, Column; Characteristic 1)

Publisher's Note Springer Nature remains neutral with regard to jurisdictional claims in published maps and institutional affiliations. 